INTERNATIONAL JOURNAI OF SYSTEMATIC BACTERIOLOGY

Vol. 17, No. 3 July 1967 pp. 263-266

Copyright 1967, Iowa State University Press

\title{
SOUS-COMITE DES PASTEURELLA, YERSINIA ET FRANCISELLA
}

Après une réunion préliminaire à Berne, en février 1966 (professeurs Knapp, Mollaret et Thal), le Sous-Comité provisoire s'est réuni à Moscou les 22 et 23 juillet 1966 durant le $\mathrm{IX}^{\circ}$ Congrès International de Microbiologie. Les membres suivants de ce Sous-Comité étaient présents: Dr. T. Burrows, Dr. G. Carter, Prof. I. V. Domaradskij, Prof. W. Knapp, Dr. N. Mair, Dr. I. Martinevskij, Prof. H.H. Mollaret, ainsi que le Prof. N.G. Olsuf'ev, invité par les membres présents à entrer dans le Sous-Comité.

Le président et le secrétaire définitifs ont été élus et la composition du Sous-Comité a été définitivement fixée (liste ci-jointe).

Le démembrement envisage du genre Pasteurella en nouveaux genres ou espèces a amené la création de six groupes de travail, respectivement consacrés aux espèces suivantes: $\left.1^{\circ}\right)$ Pasteurella pseudotuberculosis (Yersinia pseudotuberculosis, bacille de Malassez et Vignal), $2^{\circ}$ ) Pasteurella pestis (Yersinia pestis), $3^{\circ}$ ) Pasteurella multocida, $4^{\circ}$ ) Pasteurella " $X$ " (Yersinia enterocolitica), $5^{\circ}$ ) Pasteurella haemolytica, ureae etc, $6^{\circ}$ ) Pasteurella tularensis (Francis ella tularensis).

La composition de chaque groupe de travail figure dans la seconde annexe. Chaque groupe est libre de s'adjoindre des conseillers, non membres du Sous-Comité. Chaque groupe de travail devra définir, pour l'espece dont il est chargé, les propriétiés morphologiques, culturales, biochimiques, antigéniques, immunologiques, le pouvoir pathogène expérimental, la sensibilité au bactériophage et la production de bactériocine,afin d'en établir les critères d' identification et de différenciation, la taxonomie et la systématique. Outre ce programme de travail, les modalités d'échanges de souches microbiennes ont été définies.

Les membres du Sous-Comité présents à Moscou ont été unanimement d'accord pour réunir Pasteurella pseudotuberculosis et Pasteurella pestis dans le genre nouveau Yersinia et ranger Pasteurella tularensis dans le genre nouveau Francisella.

La prochaine réunion du Sous-Comité est prévue à Paris, en juillet 1967. 


\section{ANNEXE ।}

Sous-Comité des Pasteurella, Yersinia et Francisella (Subcommittee of Pasteurella, Yersinia and Erancisella)

\section{Président:}

Knapp, Prof. W., Universität Bern, Institut für Hygiene Secrétaire: und Medizinische Mikrobiologie, Bern, Suisse

Mollaret, Prof. H. H., Institut Pasteur, 25 rue du Docteur Roux, Paris $\mathrm{XV}^{\circ}$, France

\section{Membres:}

Biberstein, Prof. E. L., School of Veterinary Medicine, University of California, Davis, California 95616, U.S.A.

Burrows, Dr. T.W., Microbiological Research Establishment, Porton, near Salisbury, Wilts, Great Britain

Carter, Dr. G.R., Georgia Coastal Plain Experiment Station, Tifton, Georgia 31794, U.S.A.

Domaradskij, Prof. I. V., Institut Antipesteux, Rostov-surDon, U.R.S.S.

Frederiksen, Dr. W., Statens Seruminstitut, Regionalafdelingen, Aalborg Amtssygehus, Aalborg, Danemark

Henriksen, Prof. S. D. , Kaptein W. Wilhelmsen og frues, Bakteriologiske Institutt, Rikshospitalet, Oslo, Norvège

Lawton, Dr. W. D. , United States Army, Chemical Corps Biological Laboratories, Fort Detrick, Maryland, U.S.A.

Mair, Dr. N.S., Public Health Laboratory Service, Groby Road Hospital, Groby Road, Leicester, Grande Bretagne

Martinevskij, Dr. I. L., Institut Antipesteux, Alma-Ata, U.R.S.S.

Meyer, Prof. K. F., George William Hooper Foundation, University of California Medical Center, San Francisco, California, U.S. A.

Namioka, Dr. S., First Bacteriological Section, National Institute of Animal Health, Kodaira-City, Tokyo, Japon

Olsuf'ev, Prof. N. G., Institut Gamaleia, Moscou, U. R.S. S. Smith, Dr. J.E., Battersea-College of Technologie, 14 Falcon Road, London SW 11 , Grande Bretagne Thal, Prof. E. , Statens Veterinärmediciniska Anstalt, Stockholm 50, Suè de 
Membre ex-officio:

Seeliger, Prof. H. , Institut für Hygiene und Mikrobiologie der Universität Wurzburg, 87 Wurzburg, JosefSchneider Strasse 2, Bau 17, Allemagne

ANNEXE 2

Composition des Groupes de Travail du Sous-Comité des Pasteurella, Yersinia et Francisella (Composition of the working groups of the Subcommittee of Pasteurella, Yersinia and Francisella)

1. Pasteurella pseudotuberculosis (Pfeiffer) Topley and Wilson 1929

W. Knapp (Yersinia pseudotuberculosis W. Laston (Pfeiffer) comb. nov.) Bacille de N. Mair Malassez et Vignal.

H. H. Mollaret

E. Thal

2. Pasteurella pestis (Lehmann and Neumann) Bergey et al. 1923 (Yersinia pestis (Lehmann and

T. Burrows Neumann) van Loghem 1944. I.V. Domaradskij

W. Lawton I. Martinevskij K. F. Meyer et le Dr. Levy, non membre du Sous-Comité

3. Pasteurella multocida (Lehmann and Neumann) Rosenbusch and Merchant 1939.
E. Biberstein

W. Frederiksen

G. Carter

S. Namioka

J. Smith

4. Pasteurella haemolytica, ureae etc.
E. Biberstein
G. Carter
S. Henriksen
J. Smith

5. Pasteurella " $X$ " W. Frederiksen (Yersinia enterocolitica nom. nov.) H. H. Mollaret E. Thal 
6. Pasteurella tularensis

(Francisella tularensis (McCoy and Chapin) Dorofeer 1947).

et la Pr. Knothe (Kiel) non membre du sous-comité
K. F. Meyer

N. G. Olsuf'ev

J. Smith

H. H. Mollaret

Secretary 